was decreased in skeletal muscle but not in cardiac muscle. (Takayanagi, $\mathrm{T}$ et al. Infantile cytochromic $c$ oxidase deficiency with neonatal death. Pediatr Neurol May/June 1989; $\underline{5}: 179-81)$.

COMMENT. The differential diagnosis of neonatal respiratory distress syndrame should include mitochondrial myopathy. The diagnosis should still be considered even in the absence of ragged-red fibers in skeletal muscle.

Two further papers concerning mitochondrial myopathy appeared in the June 1989 issue of the Annals of Neurology. Shimozumi H et al established cultured myogenic cell lines that were defective in cytochrome c oxidase enzyme from a patient with mitochondrial encephalamyelopathy. Two kinds of myogenic cell lines, one with and one without defective enzymatic activity were demonstrated showing that a partial enzyme defect is the result of the cellular mosaicism in the tissue. The authors comment that these cloned cell lines provide an excellent system for clarifying the cause of mitochondrial myopathy and for investigating the genetic factors.

Sakuta $\mathrm{R}$ and Nonaka I examined muscle taken at biopsy in six patients with camplex I deficiency and one patient with the clinical characteristics of mitochondrial myopathy, encephalopathy, lactic acidosis and stroke-like episodes (MELAS). Striking abnormalities in the blood vessels were shown by electron microscopy in five patients. The authors considered that these abnormalities in small arteries might be responsible for the occasional occurence of transient cerebral ischemia causing stroke-like episodes and progressive mental deterioration in patients with mitochondrial myopathy.

\title{
MALFORMATIONS
}

\section{TETHERED SPINAL CORDS}

The diagnosis of tethered spinal cord by MRI in seven children with cutaneous lumbar hemangioma is reported from the Children's Hospital of Pittsburgh, University of Pittsburgh School of Medicine. The hemangiamas ranged in size from $4 \times 6 \mathrm{~cm}$ to $8 \times 20 \mathrm{~cm}$ and all overlapped the midline. All demonstrated tethered cords; four showed intraspinal lipamas, and two showed tight fila terminale. At surgery, all infants were found to have tethered cords and none had an intraspinal hemangioma. All patients were neurologically normal both pre and postoperatively. (Albright AL et al. Lumbar cutaneous hemangiomas as indicators of tethered spinal cords. Pediatrics June $1989 ; 83: 977-980)$.

COMMENT. The lumbar hemangiomas in these patients were large and the significance of small lesions is not known. Despite a normal neurological examination, infants or children with large lumbar cutaneous hemangiomas should be suspected of having tethered cords and magnetic resonance imaging should be obtained. If neurologic deficits 
are allowed to develop with increasing age, the likelihood of postoperative improvement is only 25-50\%. Ultrasound may be used to diagnose tethered cords but magnetic resonance is usually required for better visualization. In addition to the association with cutaneous hemangiama, tethered cord occurs with subcutaneous lipama, a hairy tuft, a prominent dimple, or a midline sinus tract or skin defect.

\section{TRAPPED VENIRICLE AND SHUNTED HYDROCEPHALUS}

Occluded fourth ventricle (trapped ventricle) is reported in eight of 47 children (17\%) receiving repeated shunt revisions for hydrocephalus at the Stritch School of Medicine, Loyola University of Chicago, Maywood, IL. The hydrocephalus was caused by intraventricular hemorrhage but the fourth ventricular enlargement developed only after shunting. Massive dilatation of the ventricle occurred in four, three developed a progressive spastic quadriparesis, and two had increased intracranial pressure with lethargy and vamiting. Two children underwent a fourth ventricular shunt; one became more alert and less quadriparetic, and the other showed gradual improvement in motor function. (Coker SB and Anderson, CL. Occluded fourth ventricle after multiple shunt revisions for hydrocephalus. Pediatrics Jume $1989 ; 83: 981-985)$.

COMMENT. Trapped ventricle following repeated shunting may be manifested by headache, lethargy, vomiting, ataxia, spastic quadriparesis, cranial nerve palsies and head tilt. This complication appears to be cammon among children with intraventricular hemorrhage who have received ventricular peritoneal shunting. Progressive fourth ventricular enlargement may be silent and diagnosis requires post shunt neural imaging and brain stem auditory evoked responses. Shunting of the fourth ventricle results in clinical improvement.

\section{TOXIC DISORDERS}

HYPERBILIRUBINEMIA AND NEURODEVEIOPMENTAL OUIOOME

The results of the Collaborative Project on Preterm and Small for Gestational Age Infants in the Netherlands, 1983, in regard to hyperbilirubinemia and neurodevelopmental outcame at two years of age are reported from the Division of Neonatology, Department of Pediatrics, University Hospital, Leiden, the Netherlands.

Children with minor and major handicaps had significantly greater maximal serum total bilirubin concentrations than children with a normal neurodevelopmental outcame $(P=0.02)$. An increase in prevalence of handicaps was found for each $50 \mathrm{mmol} / \mathrm{L}(2.9 \mathrm{mg} / \mathrm{dL})$ increase of maximal serum total bilirubin concentration. The neurological abnormalities included cerebral palsy, seizures, hearing defects as well as retinopathy of prematurity. The risk of a handicap increased by $30 \%$ for each 2.9 $\mathrm{mg} / \mathrm{dL}$ increase of maximal serum total bilirubin concentration $(P=0.02)$ suggesting a causal relationship. (van de Bor M et al. Hyperbilirubinemia 